\title{
Preparation of Immunotoxin Herceptin-Botulinum and Killing Effects on Two Breast Cancer Cell Lines
}

\author{
Saieh Hajighasemlou ${ }^{1,2}$, Mahmoud Alebouyeh ${ }^{1}$, Hossein Rastegar ${ }^{1}$, Mojgan \\ Taghizadeh Manzari ${ }^{1}$, Milad Mirmoghtadaei ${ }^{3}$, Behjat Moayedi ${ }^{4}$,Maryam \\ Ahmadzadeh $^{5}$, Farzad Parvizpour ${ }^{2}$, Behrooz Johari ${ }^{6}$, Maria Moslemi Naeini ${ }^{7}$, \\ Mohammad M Farajollahi ${ }^{7,8,9 *}$
}

\begin{abstract}
Background: Worldwide, breast cancer is the most common cancer diagnosed among women and a leading cause of cancer deaths. The age of onset in Iran has become reduced by a decade for unknown reasons. Herceptin, a humanized monoclonal antibody, is a target therapy for breast cancer cells with over expression of HER2neu receptors, but it is an expensive drug with only $20 \%$ beneficial rate of survival. This study introduces a novel approach to enhance the efficacy of this drug through immunoconjugation of the antibody to botulinum toxin. Decreasing the cost and adverse effects of the antibody were secondary goals of this study. Materials and Methods: Botulinum toxin was conjugated with Herceptin using heterobifunctional cross linkers, succinimidyl acetylthiopropionate (SATP) and sulfo-succinimidyl-4-(N-maleimidomethyl) cyclohexane-1-carboxylate (SMCC) according to the supplier's guidelines and tested on two breast cancer cell lines: SK-BR-3 and BT-474. Toxin and Herceptin were also used separately as controls. The cytotoxicity assay was also performed using the new bioconjugate on cultured cells with Alamar blue and a fluorescence plate reader. Results: Herceptin-Toxin bioconjugation significantly improved Herceptin efficacy on both breast cancer cell lines when compared to the control group. Conclusions: Toxin-Herceptin bioconjugation can be a potential candidate with increased efficiency for treating breast cancer patients with over expression of the HER2 receptor.
\end{abstract}

Keywords: Breast cancer - herceptin - botulinum toxin - HER2 - SK-BR-3 - BT-474

Asian Pac J Cancer Prev, 16 (14), 5977-5981

\section{Introduction}

Worldwide, breast cancer is the most common cancer found among women and the leading cause of deaths. According to the American Cancer Society, approximately 232,340 new of invasive cases and 39,620 deaths are expected to occur among US women in 2013. One in 8 women in the United States will develop breast cancer in her lifetime. The rate of incidence rates increased slightly among African American women; decreased among Hispanic women; and remained stable among whites, Asian Americans/Pacific Islanders, and American Indians/ Alaska natives from 2006 to 2010 (Althuis et al., 2005). Global differences in incidence rates and fluctuations in rates within a country are both affected by changes in risk factor for prevalence and secular trends in diagnosis (DeSantis et al., 2014).
The peak incidence of breast cancer in Iran is between the ages 40-50, which is a decade younger than that of the western countries (Hosseini et al., 2013). In Iran, it ranks first among cancers diagnosed in women and the fifth most common cause of death (Taghavi et al., 2012). Although the exact cause is not known, genetic and environmental factors seem to play roles.

The human epidermal growth factor receptor-2 gene C-Erb2/neu (also known as HER2/neu) encodes 1255 amino acids to a membrane glycoprotein receptor of $185 \mathrm{KD}$ from the tyrosine kinase family (Kaufmann et al., 2011). HER2 receptor regulate cell growth and differentiation through specific signal transduction pathways. HER2 gene amplification on chromosome 17 accounts for about $25 \%$ of over expressions leading to tumor growth, lengthening the $S$ phase in cell cycle, aneuploidy and down-regulation of estrogen and progesterone receptors (d'Agnano et al.,

${ }^{1}$ Iran Food and Drug Administration, ${ }^{2}$ Department of Applied Cell Sciences, School of Advanced Technologies in Medicine, Tehran University of Medical Sciences, ${ }^{3}$ Tehran University of Medical Sciences, International Campus (TUMS-IC), ${ }^{5}$ Department of Pharmaceutical Biotechnology, School of Pharmacy, Shahid Beheshti University of Medical Sciences, Tehran, Iran, ${ }^{6}$ Department of Biotechnology, Pasteur Institute of Iran, ${ }^{7}$ Cellular \& Molecular Research Centre, ${ }^{9}$ Department of Medical Biotechnology, Iran University of Medical Sciences, Tehran, ${ }^{4}$ Isfahan University of Medical Sciences, Isfahan, Iran, ${ }^{8}$ Alborz Food and Drug Laboratory, Fardis, Alborz, Iran*For correspondence: mahdiy@yahoo.com 
1996; Alshenawy, 2012).

Herceptin (Trastuzumab) is a humanized monoclonal IgG1 kappa antibody that selectively binds with high affinity to the extracellular domain of HER2 receptors with high affinity (Beuzeboc et al., 1999). Trastuzumab induces down modulation of HER2 receptor and, as a result, inhibits critical signalling pathways (i.e. ras-RafMAPK and PI3K/Akt) and blocks cell cycle progression by inducing the formation of $\mathrm{p} 27 / \mathrm{Cdk} 2$ complexes. Trastuzumab also inhibits HER2 cleavage, preceding antibody-induced receptor down modulation that in turn contribute to its antitumor activity in some tumor cells. Moreover, trastuzumab have shown to inhibits angiogenesis and induces antibody-dependent cellular cytotoxicity (Albanell et al., 2003).Immunotheraputic administration of Herceptin is a promising new approach in breast cancer treatment, complementing surgery, and other therapy such as chemotherapy, radiation and antihormonal therapy (Untch et al., 2003). Inspite of these advances Herceptin is an expensive intervention with side effects such as: cardiovascular adverse events (Tsai et al., 2014) leukopenia, hypotension, dyspnea, anemia, fatigue, headache, fever, diarrhea (Dang et al., 2010), and skin rash (Adachi et al., 2011). Nevertheless its activity is largely restricted to treatment and management of breast cancers cases with the highest level of HER2 gene amplification (Albanell et al., 2003).

Botulinum toxin is a neurotoxin produced by the bacterium Clostridium Botulinum (Yoneyama et al., 2008). To date eight serotypes of this toxin (A through $\mathrm{H})$ have been identified. The toxin endocytosed after binding to neurons and inhibits acetylcholine release via the photolytic activity of its catalytic domain (Ray, 1993). Some medical applications of Botulinum Toxin include treating children with cerebral palsy (Nezu and Iwasaki, 2013), overactive bladder(Ravindra et al., 2013), excessive sweating (Naumann et al., 2002), chronic migraine prevention of salivary stagnation after surgery of oral cavity cancer (Corradino et al., 2012) and controlling pain after mastectomy (Layeeque et al., 2004). Botulinum Toxin is also shown to induce apoptosis in T47D breast cancer cell line through caspase- 3 and -7 pathways (Bandala et al., 2013).

In this study we conjugated Herceptin to Botulinum to enhance its efficiency and possibly reducing the therapeutic dose of the drug.

\section{Materials and Methods}

\section{Herceptin preparation and activation:}

$100 \mathrm{mg}$ Herceptin was dissolved in deionized water and transferred to a dialysis bag containing $50 \mathrm{mM}$ phosphate buffer (pH: 7.5), contains 1 mM EDTA, and kept for 12 hours at $4^{\circ} \mathrm{C}$ to remove small molecules from Herceptin. $300 \mu 1$ Herceptin $(5 \mathrm{mg} / \mathrm{ml})$ was mixed with $20 \mu 1$ SATP (N-succinimidyl-S-acetylthiopropionate) solution and incubated for 60 minutes at room temperature and passed through superdex G-10 chromatography column to remove excess SAPT from antibody solution. $50 \mu 1$ Hydroxyl amine solution was also added to 500 $\mu 1$ Herceptin-SATP to protect the $-\mathrm{SH}$ groups. After 2 hours of incubation the solution was dialyzed in $50 \mathrm{mM}$ phosphate buffer (pH: 7.5), contains 1 mM EDTA, for 12 hours at $4^{\circ} \mathrm{C}$.

\section{Botulinum Toxin preparation and activation:}

$250 \mu 1$ toxin aqueous solution $(1 \mathrm{mg} / \mathrm{ml})$ was dialyzed in $0.1 \mathrm{mM}$ phosphate buffer (pH: 7.5), contains $1 \mathrm{mM}$ EDTA, for 12 hours at $4^{\circ} \mathrm{C}$ and ultra-centrifuged at $4^{\circ} \mathrm{C}$ to remove the accompanying materials.

\section{Immunotoxin preparation:}

$150 \mu \mathrm{L}$ concentrated Botulinum Toxin was added to the same volume of Herceptin-SATP (molar ratio1:1) in a microtube and the mixture was wrapped in aluminum foil and kept for 18 hours at room temperature (Li et al., 2008).

\section{High-Performance Liquid Chromatography (HPLC) Testing:}

Diol-200-8.0X500-DL20S05-5008WT column was used to confirm the bioconjugation reaction by HPLC analysis. For column preparation, $0.1 \mathrm{M}$ phosphate buffer including $0.15 \mathrm{NaCl}$ was flown through the column at least for 1 hour. Then $10 \mu 1$ of each samples was run for 50 minutes, and sample concentration was calculated by OD measurement at $280 \mathrm{~nm}$.

\section{Cell culture}

Two breast cancer cell lines; SK-BR-3 and BT474; were purchased from Pasteur Institute of Iran and cultured at standard conditions at $37^{\circ} \mathrm{C}, 95 \%$ humidity and $5 \%$. The culture medium was replaced at 48 and 72 hour intervals, to feed the cell lines and maintain the cell growth. Each cultured cell line was divided into four groups, based on the following treatments: Group 1: Cells + medium + Botulinum toxin $(20 \mu \mathrm{g} / \mathrm{ml})$; Group 2 : Cells + medium + Herceptin $(20 \mu \mathrm{g} / \mathrm{ml})$; Group 3: Cells + medium + Botulinum-Herceptin $(20 \mu \mathrm{g} / \mathrm{ml})$; Group 4 : Cells + medium

Alamar blue $(25 \mu 1)$ was added to the wells after 5 days and the fluorescence intensity was measured using a fluorescence reader at wavelengths $530 \mathrm{~nm}$ (excitation) and $605 \mathrm{~nm}$ (emission) to monitor the cell viability.

\section{Results}

HPLC procedures: The HPLC analysis of the bioconjugate preperation was performed according to the figure legends to find out various HPLC peaks for Trastuzumab, the toxin and the bioconjugate (immunotoxin) to ensure proper conjugation of two components (antibody and toxin).

As it is shown in figure 1, the second peak (Retention Time $=28.542 \mathrm{~min}$ ) corresponds to the toxin. Peak 1 represents oligomeric form of the toxin, peak 2 corresponds to the monomeric form of the toxin, Peaks 3 and 4 correspond to the small molecules in the toxin buffer matrix.

Figure 2 shows the chromatogram of Herceptin, in which only one peak can be seen to respect the antibody monomer without any other contaminations or component.

Figure 3 shows the HPLC Analysis of the conjugation 


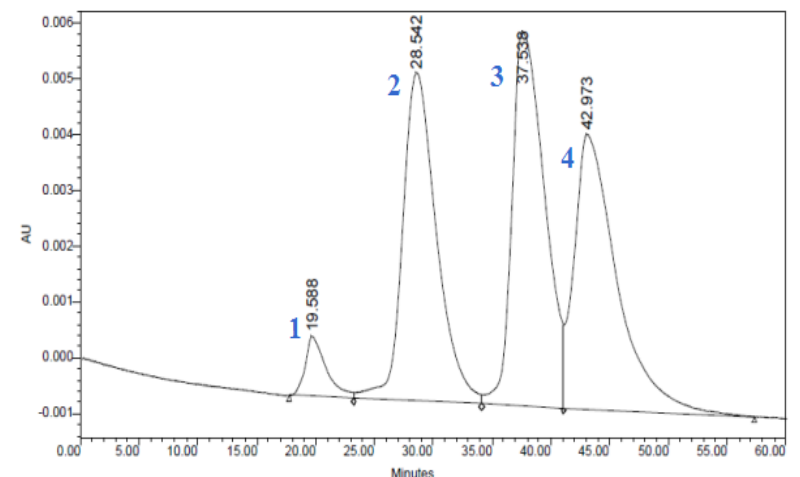

Figure 1. Chromatogram of the Toxin; Peak 1: Oligomeric Protein; peak 2: Monomeric Protein; Peak 3\&4: Small Molecules. Column: YMC-Pack Diol-2008.0X500-DL20S05-5008WT, Mobile phase: 0.1M Phosphate buffered saline (PBS) with $0.15 \mathrm{M} \mathrm{NaCl}$. Injection volume: $10 \mu 1$. Runtime: 50 minutes. Detector: Ultraviolet Wavelength: $280 \mathrm{~nm}$. Flow rate: $0.5 \mathrm{ml} / \mathrm{min}$

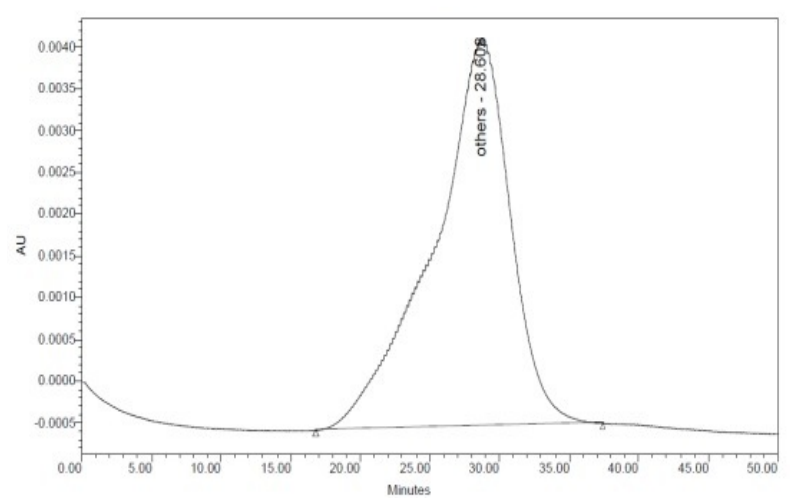

Figure 2. HPLC chromatograph of Herceptin: the peak Corresponds to Purified Herceptin (Retention Time: 28.608min). All the Chromatographic Conditions were the Same as Figure 1

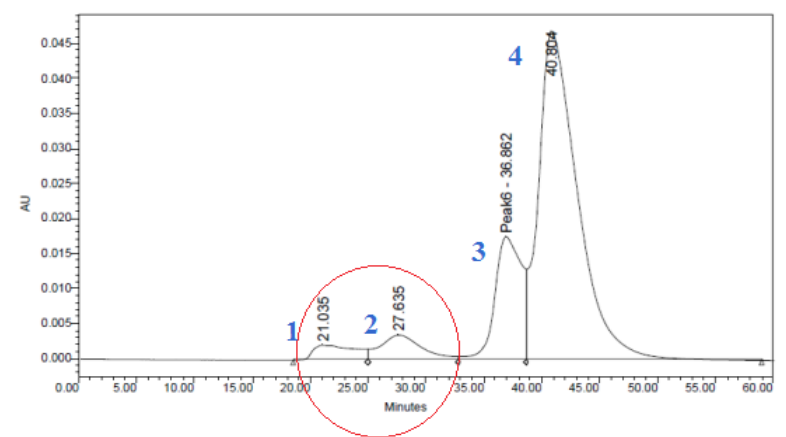

Figure 3. HPLC chromatograph of the Bioconjugate; Peaks 1\&2: Toxin-antibody Bioconjugate; Peaks 3\&4: Small Bounded Co-molecules

reaction mixture. Chromatographic conditions were the same as figure 1 . The first and second peaks correspond to the bioconjugat molecules at two different molecular ratio. The conjugation precedures combine 1-3 molecules of toxin per antibody molecules. The two peaks here, coresponds to 1-3 bioconjugate molecules based on the molecular ratio of both molecules, and the 3rd and 4th peaks corespond to non-conjugated biomolecules.

\section{Cell culture results:}

We observed a substantial reduction in the number
In vitro Enhancement of Immunotoxin Herceptin Killing Effects

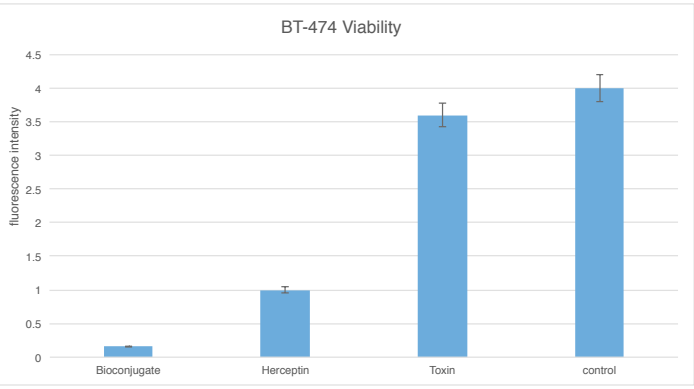

Figure 4. Fluorescence Intensity (average +/- standard deviation) of BT-474 Cell Line in Different Groups shows Significant Reduction $(94.96 \%)$ in Cell Viability in the Bioconjugate Group $(\mathbf{P}<0.001)$

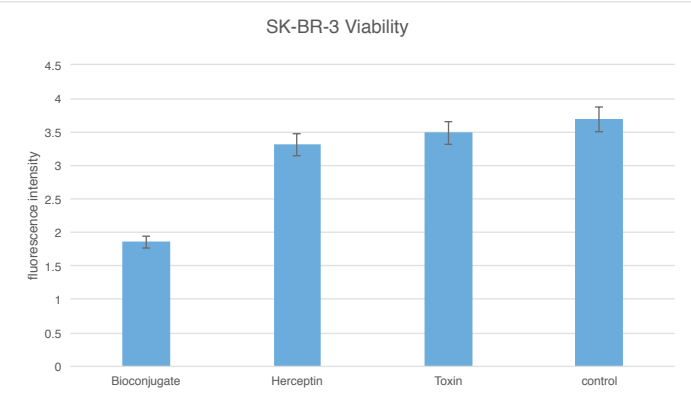

Figure 5. Fluorescence Intensity (Average +/-Standard deviation) of SK-BR-3 Cell Line in Different Groups Shows Significant Reduction $(\mathbf{9 5 . 8 \%})$ Cell Viability in the Bioconjugate Group $(P<0.001)$

of cells $(94.96 \%$ and $95.8 \%$ in BT-474 and SK-BR-3 respectively) in both cell lines when treated with the bioconjugate when compared to the untreated control. Herceptin alone showed higher efficacy on BT-474 cell line (figure 4) compared to a condition where the antibody tested on SK-BR-3 cells (figure 5). Decrease in cell viability was also more dramatic when BT- 474 cells treated with the bioconjugate.

\section{Discussion}

Resistance to Herceptin therapy has been documented in majority of patients with HER2 over expression (Ahmad et al., 2014). Only $20 \%$ of patients with amplified HER $2 /$ neu response to Herceptin. Moreover; A cardio toxicity profile has been described in more than 10 percent of the patients who used Herceptin, thus its dosage needs to be carefully adjusted and tailored to each patient, especially in elderly patients with cardiovascular problems (Sparano, 2001).

The new bioconjugate (immunotoxin) showed significant reduction in the viability of BT-474 and SKBR-3 cell lines compared to both Herceptin and botulinum toxin individually. This result indicates significant synergistic reduction effect of bioconjugated drug on cancer cells viability $94.96 \%$ and $95.8 \%$ in BT-474 and SK-BR-3 respectively.

The efficacy of conventional cytotoxic medications is directly related to the ratio of dividing tumor cells to healthy cells, as they are distributed to all tissues through blood due to their low molecular weight and 
lack of specificity. This will in turn lead to adverse side effects and resistance. In the last decade recombinant biopharmaceuticals have contributed in many novel therapeutics with higher specificity which renders them more effective and more tolerable (Olver, 2008). Researchers attach cytotoxins to macromolecules (e.g. antibodies, serum proteins, lectins, peptides, growth factors and synthetic polymers) to enhance their efficacy and reduce the unwanted effects. Antibodies play an important role in cancer therapies, their activity however, is short lived (Meyer et al., 2014).

Immunotoxin consist of a toxin attached to an antibody or a growth factor with a specific target to enter the cytoplasm and to mediate their effects on the normal cells (Avila et al., 2007; Singh et al., 2007). The bioconjugate improves the therapeutic profile of the antibody by increasing its tissue distribution, reducing its side effect on the healthy cells and increasing the half-life of the medication. In vivo experiments and clinical trials have established the effectiveness of new bioconjugates (Dao et al., 2012).

The first effective immunotoxins were plant derived toxins like ricin that could inhibit protein synthesis via inhibiting ribosomal RNA (Chan et al., 2004) and vascular injuries associated with this drug ameliorated by genetic modifications of Ricin A chain (Mohanraj and Ramakrishnan, 1995). Other herbal cytotoxins include: Saporin, Gelonin and Poke weed. Immunotoxins can inhibit cell growth through inhibiting protein synthesis and inducing apoptosis. (Houchins, 2000) Anti-c-Met/ PE38KDEL for instance, targets stomach cells in this manner (Wei et al., 2011).

Breast cancer is the most prevalent cancer among women worldwide and the most frequent malignancy among Iranian women over the past few decades (Afsharfard et al., 2013), with an increasing trend. Herceptin (Trastuzumab) is a monoclonal antibody against the extracellular domain of HER2 with humanized complementary-determining regions (CDRs) of monoclonal antibody 4D5 (Albanell and Baselga, 1999). Herceptin therapy adjuvant to chemotherapy has shown to increase therapeutic response and survival in HER2/ neu positive patients (Perez, 2001)

Barok et al investigated the effect of a new conjugate trastuzumab-DM1 on four gastric cancer cell lines (N-87, OE-19, SNU-216 and MKN-7). N-87 and OE-19 showed greater sensitivity compared to Herceptin alone. MKN-7 showed moderate response while a limited efficacy was observed in SNU-216. The study was performed in in-vitro setting where its analysis supported our data (Barok et al., 2011).

Lattová et al used Herceptin in combination with Lipoplex mixed with plasmid DNA against Herceptin resistant cell lines and showed a positive response when measured by trypan blue after 72 hours (Lattova et al., 2010). In this study we used Alamar blue which is more sensitive than trypan blue.

Similar in-vitro and in-vivo experiments have confirmed the efficacy of immunotoxins in treatment of different types of malignancies. Moreover we can isolate the light chain of the toxin responsible for its protease activity and utilize the Herceptin to function as the toxin heavy chain for cell targeting and endocytosis.

Finally, this study demonstrated an increase in the efficacy of Herceptin when bound to Botulinum toxin. This could in turn lead to a lower dose of administration and therefore lower rates of adverse side effects.

For further study, in animal model, the L-Chain of the Botulinum Toxin needs to be separated from cell-bind domain and conjugated into antibody in order to prevent destructive effect on normal cells.

\section{Acknowledgements}

We would like to thank all our study participants for their commitment to this study. Financial support was provided by Iran University of Medical Science and Iran Food and Drug Organization (IR.FDA).

\section{References}

Adachi S, Yoshimura T, Matsuoka T, et al (2011). [Appearance of skin and nail toxicity in patients with breast cancer who underwent trastuzumab-containing chemotherapy]. Gan To Kagaku Ryoho, 38, 1453-6.

Afsharfard A, Mozaffar M, Orang E, et al (2013). Trends in epidemiology, clinical and histopathological characteristics of breast cancer in Iran: results of a 17 year study. Asian Pac J Cancer Prev, 14, 6905-11.

Ahmad S, Gupta S, Kumar R, et al (2014). Herceptin resistance database for understanding mechanism of resistance in breast cancer patients. Sci Rep, 4, 4483.

Albanell J, Baselga J (1999). Trastuzumab, a humanized antiHER2 monoclonal antibody, for the treatment of breast cancer. Drugs Today (Barc), 35, 931-46.

Albanell J, Codony J, Rovira A, et al (2003). Mechanism of action of anti-HER 2 monoclonal antibodies: scientific update on trastuzumab and 2C4. Adv Exp Med Biol, 532, 253-68.

Alshenawy HA (2012). Prevalence of androgen receptors in invasive breast carcinoma and its relation with estrogen receptor, progesterone receptor and Her2/neu expression. $J$ Egypt Natl Canc Inst, 24, 77-83.

Althuis MD, Dozier JM, Anderson WF, et al (2005). Global trends in breast cancer incidence and mortality 1973-1997. Int J Epidemiol, 34, 405-12.

Avila AD, Calderon CF, Perez RM, et al (2007). Construction of an immunotoxin by linking a monoclonal antibody against the human epidermal growth factor receptor and a hemolytic toxin. Biol Res, 40, 173-83.

Bandala C, Perez-Santos JL, Lara-Padilla E, et al (2013). Effect of botulinum toxin A on proliferation and apoptosis in the T47D breast cancer cell line. Asian Pac J Cancer Prev, 14, 891-4.

Barok M, Tanner M, Koninki K, et al (2011). Trastuzumab-DM1 is highly effective in preclinical models of HER2-positive gastric cancer. Cancer Lett, 306, 171-9.

Beuzeboc P, Scholl S, Garau XS, et al (1999). [Herceptin, a monoclonal humanized antibody anti-HER2: a major therapeutic progress in breast cancers overexpressing this oncogene?]. Bull Cancer, 86, 544-9.

Chan YL, Correll CC, Wool IG (2004). The location and the significance of a cross-link between the sarcin/ricin domain of ribosomal RNA and the elongation factor-G. J Mol Biol, 337, 263-72.

Corradino B, Di Lorenzo S, Moschella F (2012). Botulinum toxin A for oral cavity cancer patients: in microsurgical patients 
BTX injections in major salivary glands temporarily reduce salivary production and the risk of local complications related to saliva stagnation. Toxins (Basel), 4, 956-61.

d'Agnano I, Bucci B, Mottolese M, et al (1996). DNA ploidy, cell kinetics, and epidermal growth factor receptor and HER2/neu oncoprotein expression in primary operable breast cancer. Ann N Y Acad Sci, 784, 472-81.

Dang C, Lin N, Moy B, et al (2010). Dose-dense doxorubicin and cyclophosphamide followed by weekly paclitaxel with trastuzumab and lapatinib in HER2/neu-overexpressed/ amplified breast cancer is not feasible because of excessive diarrhea. J Clin Oncol, 28, 2982-8.

Dao KL, Sawant RR, Hendricks JA, et al (2012). Design, synthesis, and initial biological evaluation of a steroidal anti-estrogen-doxorubicin bioconjugate for targeting estrogen receptor-positive breast cancer cells. Bioconjug Chem, 23, 785-95.

DeSantis C, Ma J, Bryan L, et al (2014). Breast cancer statistics, 2013. CA Cancer J Clin, 64, 52-62.

Hosseini MS, Arab M, Nemati Honar B, et al (2013). Age - specific incidence rate change at breast Cancer and its different histopathologic subtypes in Iran and Western countries. Pak J Med Sci, 29, 1354-7.

Houchins JP (2000). Immunotoxin-induced apoptosis. Stem Cells, 18, 384-5.

Kaufmann R, Muller P, Hildenbrand G, et al (2011). Analysis of Her2/neu membrane protein clusters in different types of breast cancer cells using localization microscopy.J Microsc, 242, 46-54.

Lattova E, Tomanek B, Bartusik D, et al (2010). N-glycomic changes in human breast carcinoma $\mathrm{MCF}-7$ and T-lymphoblastoid cells after treatment with herceptin and herceptin/Lipoplex. J Proteome Res, 9, 1533-40.

Layeeque R, Hochberg J, Siegel E, et al (2004). Botulinum toxin infiltration for pain control after mastectomy and expander reconstruction. Ann Surg, 240, 608-13.

Li LX, Tang YM, Zhang HZ, et al (2008). [Preparation of the immunotoxin 2E8-norcantharidin and its targeting killing effect in vitro]. Zhonghua Er Ke Za Zhi, 46, 493-7.

Meyer S, Leusen JH, Boross P (2014). Regulation of complement and modulation of its activity in monoclonal antibody therapy of cancer. MAbs, 6 .

Mohanraj D, Ramakrishnan S (1995). Cytotoxic effects of ricin without an interchain disulfide bond: genetic modification and chemical crosslinking studies. Biochim Biophys Acta, 1243, 399-406.

Naumann MK, Hamm H, Lowe NJ (2002). Effect of botulinum toxin type A on quality of life measures in patients with excessive axillary sweating: a randomized controlled trial. Br J Dermatol, 147, 1218-26.

Nezu A, Iwasaki N (2013). [Botulinum toxin in the treatment of cerebral palsy in childhood: the domesteic expert opinions and the role of pediatric neurologists in the management for severely handicapped children]. No To Hattatsu, 45, 147-9.

Olver IN (2008). Trastuzumab as the lead monoclonal antibody in advanced breast cancer: choosing which patient and when. Future Oncol, 4, 125-31.

Perez EA (2001). The role of adjuvant monoclonal antibody therapy for breast cancer: rationale and new studies. Curr Oncol Rep, 3, 516-22.

Ravindra P, Jackson BL, Parkinson RJ (2013). Botulinum toxin type A for the treatment of non-neurogenic overactive bladder: does using onabotulinumtoxinA $(\operatorname{Botox}((\mathrm{R})))$ or abobotulinumtoxinA $(\operatorname{Dysport}((\mathrm{R})))$ make a difference? BJU Int, 112, 94-9.

Ray P (1993). Botulinum toxin A inhibits acetylcholine release from cultured neurons in vitro. In Vitro Cell Dev Biol Anim,
29, 456-60.

Singh R, Samant U, Hyland S, et al (2007). Target-specific cytotoxic activity of recombinant immunotoxin $\mathrm{scFv}(\mathrm{MUC1})$ ETA on breast carcinoma cells and primary breast tumors. Mol Cancer Ther, 6, 562-9.

Sparano JA (2001). Cardiac toxicity of trastuzumab (Herceptin): implications for the design of adjuvant trials. Semin Oncol, 28, 20-7.

Taghavi A, Fazeli Z, Vahedi M, et al (2012). Increased trend of breast cancer mortality in Iran. Asian Pac J Cancer Prev, 13, 367-70.

Tsai HT, Isaacs C, Fu AZ, et al (2014). Risk of cardiovascular adverse events from trastuzumab (Herceptin $((\mathrm{R})))$ in elderly persons with breast cancer: a population-based study. Breast Cancer Res Treat, 144, 163-70.

Untch M, Ditsch N, Hermelink K (2003). Immunotherapy: new options in breast cancer treatment. Expert Rev Anticancer Ther, 3, 403-8.

Wei X, Juan ZX, Min FX, et al (2011). Recombinant immunotoxin anti-c-Met/PE38KDEL inhibits proliferation and promotes apoptosis of gastric cancer cells. J Exp Clin Cancer Res, 30, 67.

Yoneyama T, Miyata K, Chikai T, et al (2008). Clostridium botulinum serotype $\mathrm{D}$ neurotoxin and toxin complex bind to bovine aortic endothelial cells via sialic acid. FEMS Immunol Med Microbiol, 54, 290-8. 